\title{
Necrotizing Sarcoid Granulomatosis with Natural Resolution after a Surgical Lung Biopsy
}

\author{
Takayuki Shibata ${ }^{1}$, Koichiro Takahashi ${ }^{2}$, Masaru Uchida ${ }^{1}$, Fumio Yamasaki ${ }^{3}$, \\ Michihiro Kawashima ${ }^{2}$ and Naoko Sueoka-Aragane ${ }^{2}$
}

\begin{abstract}
:
Necrotizing sarcoid granulomatosis (NSG) is a rare disease that is diagnosed based on pathological findings. We herein report the case of a 27 -year-old man who had multiple nodular shadows in bilateral lung fields on chest radiography and elevated levels of C-reactive protein (CRP). The pathological evaluation of a lung biopsy specimen showed the infiltration of lymphocytes, granulomas with necrosis and granulomatous angiitis. He was therefore diagnosed with NSG. He has been followed without treatment, as his fever and CRP levels decreased immediately after the surgical lung biopsy. Thereafter, the pulmonary nodular shadows gradually recovered without any treatment within a few months. Our experience suggests the possibility that surgical invasion might trigger an improvement in disease activity.
\end{abstract}

Key words: necrotizing sarcoid granulomatosis, multiple nodular shadows, natural recovery, surgical lung biopsy

(Intern Med 57: 1625-1629, 2018)

(DOI: 10.2169/internalmedicine.9813-17)

\section{Introduction}

Necrotizing sarcoid granulomatosis (NSG) is a rare systemic disease that is characterized by sarcoid-like granulomas, vasculitis and variable degrees of necrosis (1). The reported clinical features include pulmonary symptoms, such as cough, dyspnea and chest pain, and extra-pulmonary symptoms, including a fever, general fatigue and weight loss $(2,3)$. However, $25 \%$ of patients with NSG are asymptomatic (4). The pathogenesis of NSG is not fully understood; it is speculated that NSG involves systemic inflammation that causes granulomas not only in the respiratory system but also in other organs (5). The prognosis of NSG has been reported to be good in previous studies $(2,4)$. Most NSG patients who have systemic symptoms, such as a fever or signs of multiple organ involvement, are treated with corticosteroids (6-8). However, some patients have recovered naturally without any treatment (2).

We herein report a rare patient with NSG who recovered from his symptoms and pulmonary nodular shadows after a surgical lung biopsy.

\section{Case Report}

A 27-year-old Japanese man visited our hospital with complaints of cough, sputum and a fever. Although he had been diagnosed with bronchial asthma during childhood, he had had no symptoms in the last 15 years. He had a 7-packyear smoking history. He had no history of dust exposure. Four weeks before admission, he had developed symptoms of cough and a low-grade fever, for which he was treated with clarithromycin for 5 days due to a diagnosis of acute bronchitis. Thereafter, his symptoms of productive cough and a fever gradually diminished. However, he developed left-sided chest pain that worsened with inspiration, which lasted for a week before admission.

On admission, his body temperature was $38.3{ }^{\circ} \mathrm{C}$. The findings on a physical examination, including an assessment of consciousness, chest auscultation, joints and skin, were

\footnotetext{
${ }^{1}$ Division of Respiratory Medicine, Japan Community Health care Organization Saga Central Hospital, Japan, ${ }^{2}$ Division of Hematology, Respiratory Medicine and Oncology, Department of Internal Medicine, Faculty of Medicine, Saga University, Japan and ${ }^{3}$ Division of Pathology, Japan Community Health care Organization Saga Central Hospital, Japan

Received: July 14, 2017; Accepted: October 10, 2017; Advance Publication by J-STAGE: January 11, 2018

Correspondence to Dr. Koichiro Takahashi, takahak@cc.saga-u.ac.jp
} 
(A)

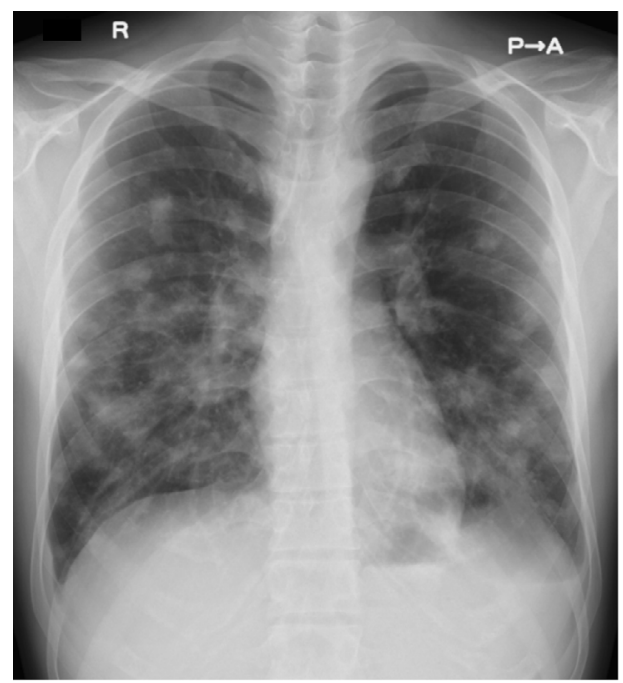

(B)
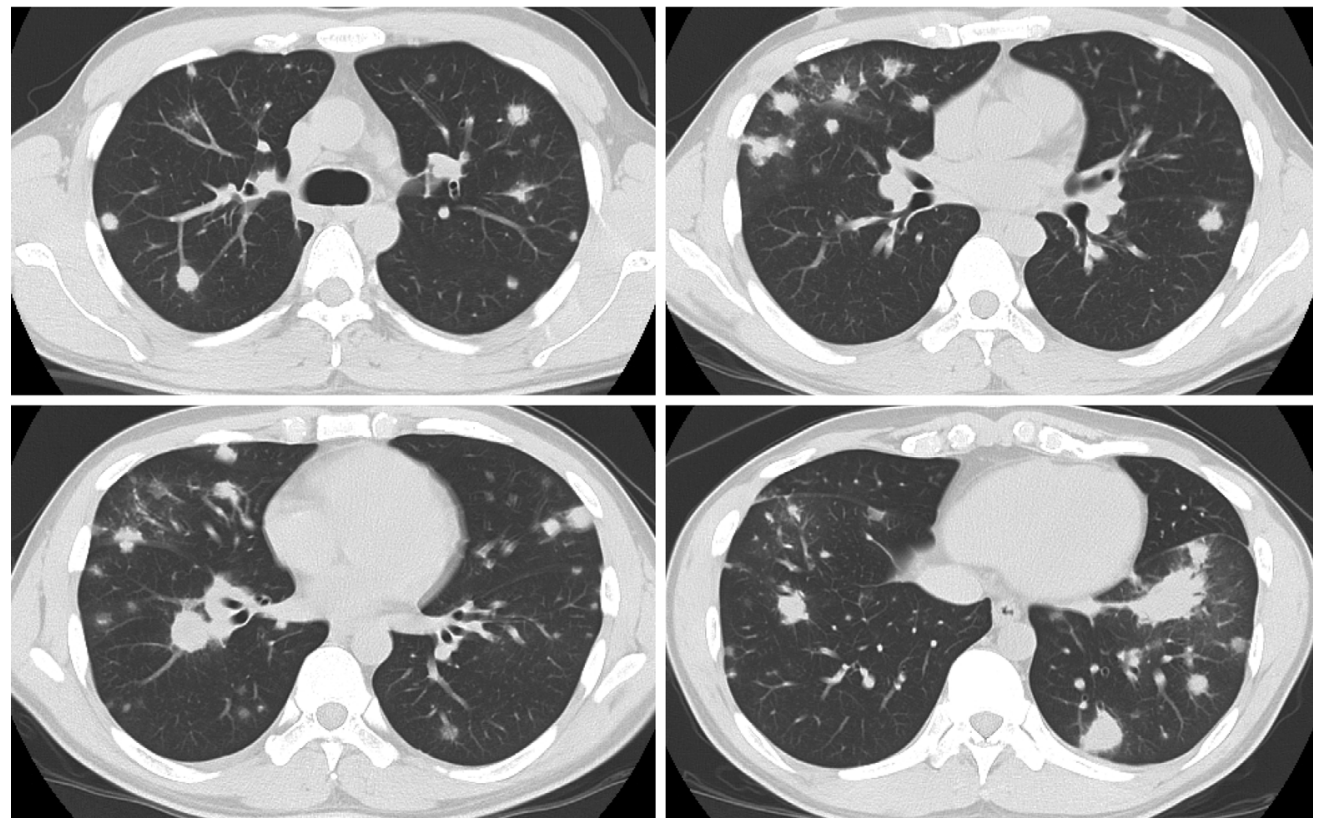

Figure 1. Findings of chest radiography and computed tomography at admission. Chest radiography showing multiple nodular shadows in both lung fields and pulmonary infiltration in the left lower lung field. (B) Chest CT showing multiple nodular shadows in the bilateral lung fields. The nodular shadows had irregular margins, and some of them appeared to be infiltration.

normal. His laboratory findings on admission revealed the following: white blood cell count $11.5 \times 10^{3} / \mu \mathrm{L}$ with $77.3 \%$ neutrophils, hemoglobin $15.2 \mathrm{~g} / \mathrm{dL}$, aspartate aminotransferase (AST) $92 \mathrm{U} / \mathrm{L}$, alanine aminotransferase (ALT) 185 U/L, lactate dehydrogenase (LDH) 203 U/L, gammaglutamyl transferase ( $\gamma$-GTP) $424 \mathrm{U} / \mathrm{L}$, alkaline phosphatase (ALP) 1,146 U/L, and C-reactive protein (CRP) $14.3 \mathrm{mg} / \mathrm{dL}$, all of which were elevated. Direct bilirubin, indirect bilirubin, platelet count, coagulation profile, angiotensinconverting enzyme (ACE), anti-myeloperoxidase (MPO)-anti neutrophil cytoplasmic antibody (ANCA), anti-serine protease3 (PR3)-ANCA, and other biochemical tests and urine studies were within the normal range. The results of an arterial blood gas analysis while breathing room air were as follows: partial pressure oxygen $\left(\mathrm{PO}_{2}\right) 82.4$ torr, carbon dioxide partial pressure $\left(\mathrm{PCO}_{2}\right) 39.6$ torr, $\mathrm{pH} 7.411$, and hydrogen- carbonate $\left(\mathrm{HCO}_{3}\right) 24.3 \mathrm{mEq} / \mathrm{L}$.

Chest radiography showed multiple nodular shadows in bilateral lung fields and segmental infiltration in the lower lung fields bilaterally (Fig. 1A). Chest computed tomography (CT) showed multiple nodular shadows in both lung fields (Fig. 1B). These shadows were seen to have irregular margins and were distributed in the sub-pleural and peribronchovascular areas. Furthermore, some of them appeared to be infiltration, although there was no cavity formation. A sputum culture examination did not reveal any bacteria, including acid-fast bacilli. Bronchoscopy and bronchoalveolar lavage (BAL) of the right middle lobe bronchus (B4) were performed using $100 \mathrm{~mL}$ saline solution, and the recovery rate was $60 \%$. An examination revealed a marked increase in the number of total cells $\left(7.7 \times 10^{6} / \mathrm{mL}\right)$ and lymphocytes $(43.5 \%)$ in the lavage fluid, while the ratio of $\mathrm{CD} 4 / \mathrm{CD} 8$ was 

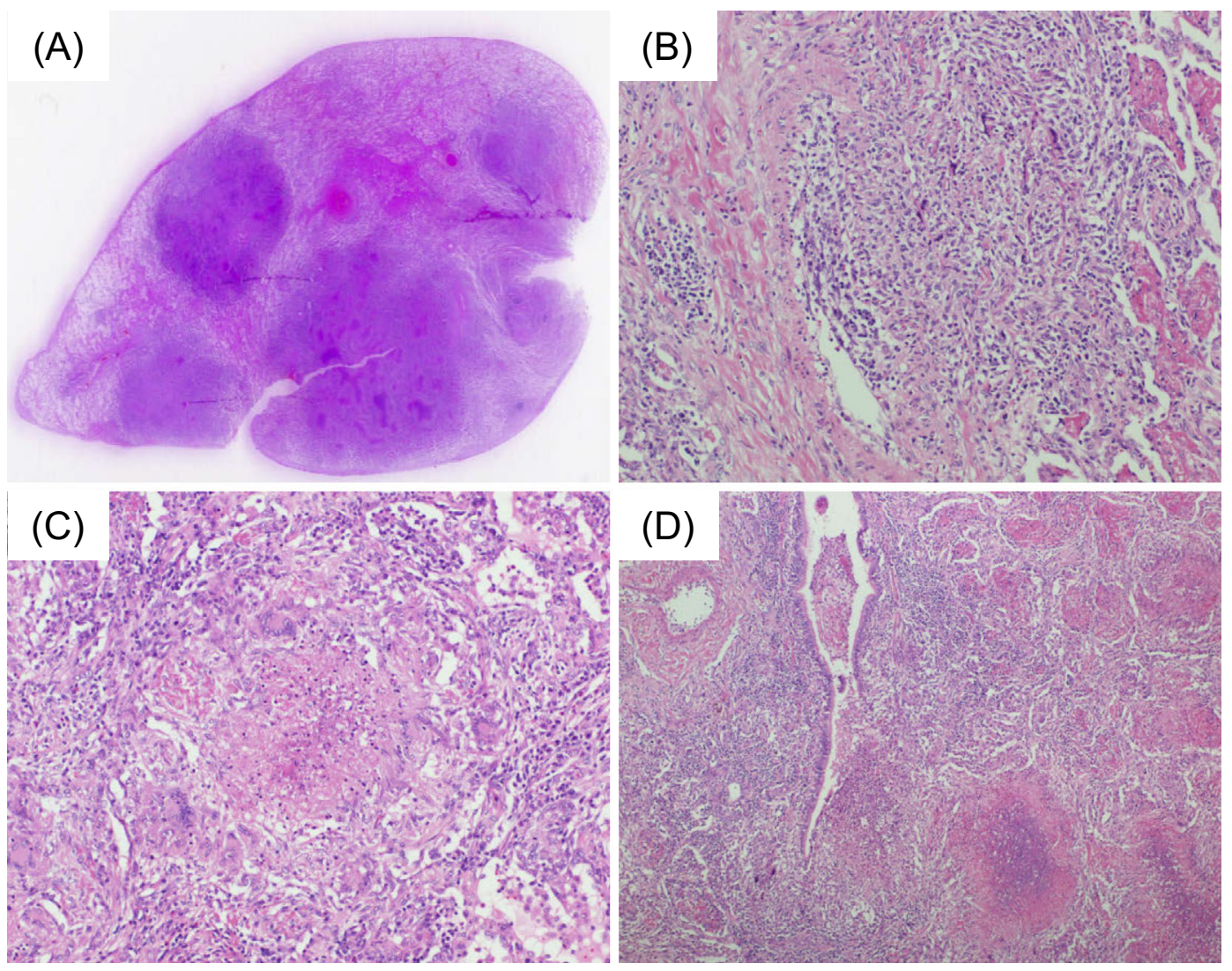

Figure 2. Pathological findings at the lung biopsy. (A) An evaluation of the biopsy specimen using loupe images revealed multiple granulomas. (B) The pathological findings of the lung showing granulomatous vasculitis (Hematoxylin and Eosin $(H \& E)$ staining, $\times 400),(C)$ granulomas with giant cells and necrosis $(H \& E$ staining, $\times 400)$ and (D) lung showing granulomatous bronchiolitis (H\&E staining, $\times 100)$.

within the normal range, at 2.27 .

A histological evaluation of a transbronchial lung biopsy showed the infiltration of lymphocytes and macrophages in the bronchus and alveoli. Subsequently, lung biopsies of the right upper lobe and right lower lobe were performed using video-assisted thoracic surgery. A histological evaluation of the biopsy specimen demonstrated infiltration of lymphocytes and granulomas with necrosis (Fig. 2A and B), granulomatous angiitis (Fig. 2C) and granulomatous bronchiolitis (Fig. 2D). There were no findings suggestive of mycosis or acid-fast bacillus infection. Based on these results, the patient was diagnosed with NSG. We decided to follow him up without any treatment, as his fever was relieved immediately after the lung biopsy.

Two weeks after the lung biopsy, chest radiography revealed an improvement in the nodular shadows. Chest radiography and CT at six months after the diagnosis showed significant improvement of the pulmonary nodular shadows (Fig. 3). This was accompanied by improvement not only in the symptoms of cough and a fever but also in the levels of AST, ALT, LDH and CRP, which returned to their normal ranges. Since then, he has been carefully followed up. At one year after the diagnosis, he still has not developed any recurrence.

\section{Discussion}

We herein report an adult patient with NSG that resolved naturally over a few months without any treatment. NSG is a rare disease, with only 200 reported cases $(2-4,9)$. The pathogenesis of NSG is still unclear. The pathological findings suggest that an inflammatory cell-mediated response, such as by lymphocytes and plasma cells, results in granuloma formation (1). Some have suggested that there is no involvement of exogenous factors, including bacteria, in this process (10).

The clinical features of NSG include pulmonary and extra-pulmonary symptoms, such as a fever, general fatigue and weight loss, although there are no specific features pathognomonic of NSG $(2,4)$. Our patient had both pulmonary and extra-pulmonary symptoms.

The most frequent radiologic manifestations of NSG are multiple, bilateral pulmonary nodules with irregular margins (11). Solitary nodules or a mass or infiltration shadows with a subpleural and bronchovascular distribution are also occasionally seen (2). Hilar or mediastinal lymphadenopathies are less common findings in NSG (33\%) than in nodular sarcoidosis (85\%) (4). Recently, fluorodeoxyglucose (FDG)-positron emission tomography (PET) has been re- 


\section{(A)}

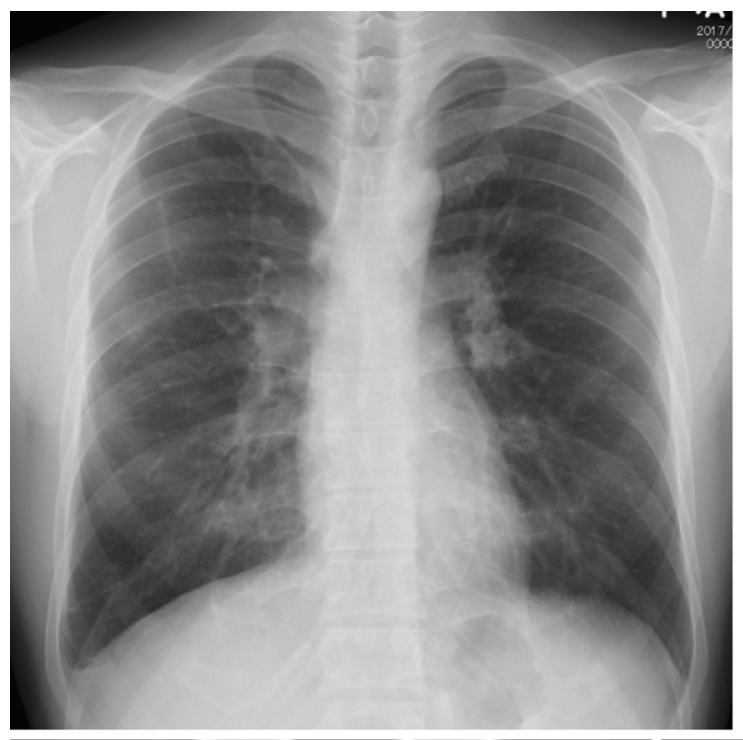

(B)
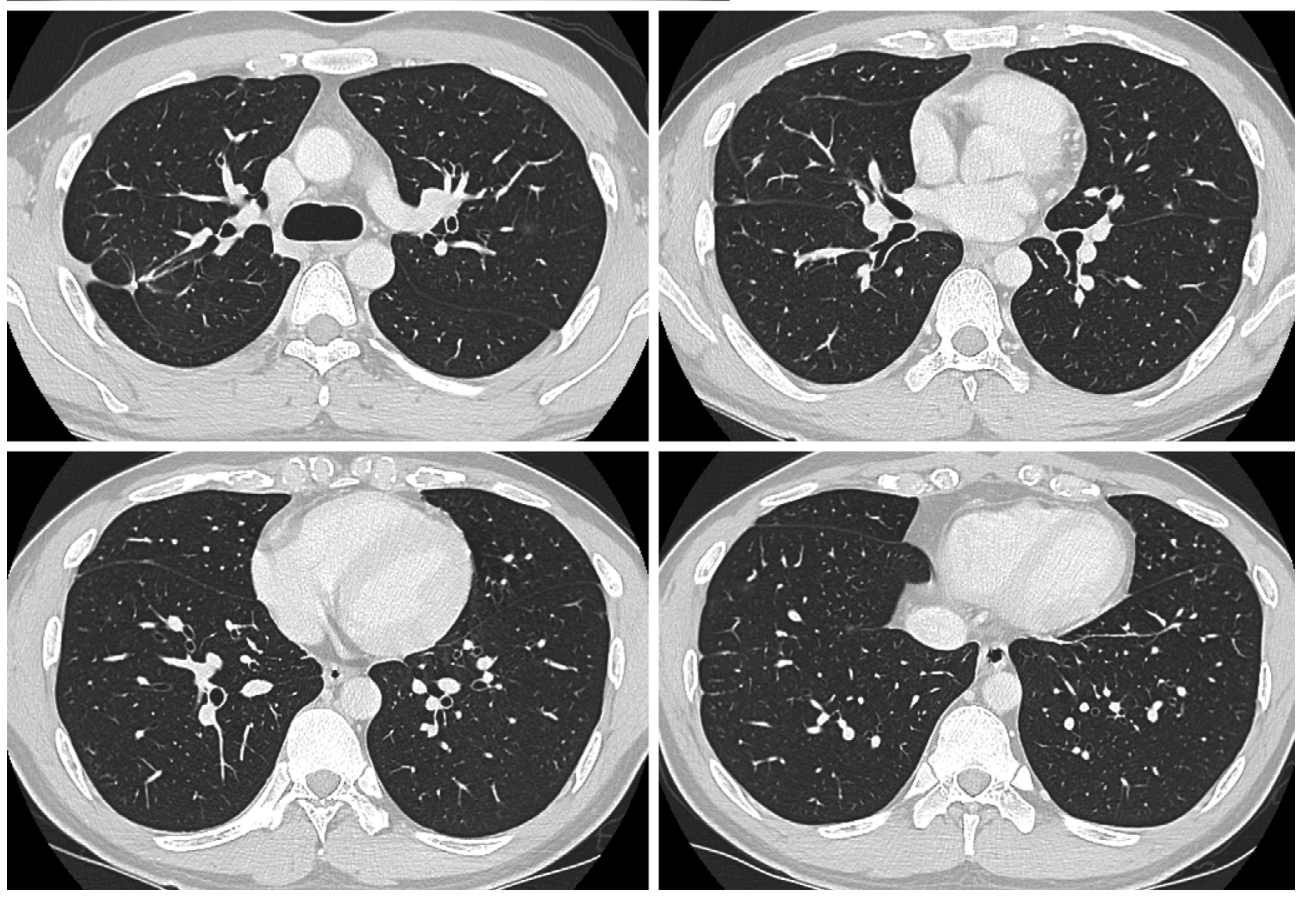

Figure 3. Findings of chest radiography at six months after the diagnosis. Chest radiography and (B) chest CT at six months after the diagnosis showed improvement in the pulmonary nodular shadows.

ported as useful for determining the biopsy site to diagnose NSG $(6,12)$.

More than $80 \%$ of the previously reported NSG patients had extra-pulmonary manifestations, including arthralgia, ocular symptoms, sicca syndrome, neurological symptoms and skin and liver involvement $(2,4)$. Our case showed elevated liver enzymes and biliary tract-associated enzymes with normal bilirubin on admission. CT of the liver, however, revealed no abnormalities, such as nodular shadows. Since the liver enzymes normalized naturally without any treatment, they were probably related to liver involvement of NSG.

The pathological features of NSG are characterized by granulomatous angiitis, granuloma with necrosis and giant cell infiltrations. Several types of vascular lesions have been observed (1). The angiitis generally consists of intramural granulomas or diffuse lymphocytic and plasma cell infiltration that is more or less confined to the vessel walls (10). Giant cell arteritis is less frequent. The extent of coagulative necrosis varies considerably, from small punctate foci in angiocentric granulomas to large extravascular confluent zones (13). In some cases, it is difficult to distinguish nodular sarcoidosis from NSG, although the existence of vasculitis is an essential finding of NSG $(4,13)$. Thus, a biopsy is essential for the diagnosis of NSG, since, along with the other usual pathological findings, the presence of vasculitis is a key feature distinguishing it from other granulomas.

Treatment of NSG mainly consists of corticosteroids. Quaden et al. reviewed 14 patients with NSG and found that $5(35.7 \%)$ initially received corticosteroids, with doses of 
prednisolone between 0.5 and $1.0 \mathrm{mg} / \mathrm{kg} / \mathrm{day}(2)$. Four patients $(28.6 \%)$ with solitary nodules underwent surgical resection; the remaining 5 patients $(35.7 \%)$ received no treatment, but their pulmonary lesions diminished in several months (2). The pulmonary lesions in the present case started to improve immediately after the surgical biopsy. Anesthesia and surgical intervention are known to induce not only the release of cytokines, including tumor necrosis factor (TNF) $\alpha$, interleukin (IL)-1 $\beta$ and IL-6, but also the production of hormones, including corticosteroids $(14,15)$. For example, sarcoidosis symptoms are known to be relieved during pregnancy and to worsen after childbirth (16). The activity of NSG may therefore improve under the influence of endogenous steroid hormones.

Previous reports have described the relapse of NSG despite initial improvement with corticosteroids. The interval between treatment and relapse was previously reported as 32 months (range 24-43 months), although there were no definitive predictive features or markers suggestive of relapse (2). Therefore, NSG patients should be followed carefully for several years.

In conclusion, we report a rare case of NSG in a patient who naturally recovered from his symptoms and pulmonary nodular shadows after a surgical lung biopsy. Surgical invasion may have triggered an improvement in the disease activity. Physicians should consider NSG in the differential diagnosis of patients with multiple pulmonary nodular shadows and should proactively perform a pathology examination.

Written informed consent was obtained from the patient for the publication of this case report.

The authors state that they have no Conflict of Interest (COI).

\section{References}

1. Liebow AA. The J. Burns Amberson lecture-pulmonary angiitis and granulomatosis. Am Rev Respir Dis 108: 1-18, 1973.

2. Quaden C, Tillie-Leblond I, Delobbe A, et al. Necrotising sarcoid granulomatosis: clinical, functional, endoscopical and radiographical evaluations. Eur Respir J 26: 778-785, 2005.

3. Churg A, Carrington CB, Gupta R. Necrotizing sarcoid granulo- matosis. Chest 76: 406-413, 1979

4. Rosen Y. Four decades of necrotizing sarcoid granulomatosis: what do we know now? Arch Pathol Lab Med 139: 252-262, 2015.

5. Yeboah J, Afkhami M, Lee C, Sharma OP. Necrotizing sarcoid granulomatosis. Curr Opin Pulm Med 18: 493-498, 2012.

6. McArdle DJT, McArdle JP, Jessup P, Harle RA, Parker S. Necrotizing sarcoid granulomatosis: clinico-radio-pathologic diagnosis. Am J Med 130: e283-e286, 2017.

7. Dykhuizen RS, Smith CC, Kennedy MM, et al. Necrotizing sarcoid granulomatosis with extrapulmonary involvement. Eur Respir J 10: 245-247, 1997.

8. Huang $\mathrm{H}, \mathrm{Li} \mathrm{C}$, Bai $\mathrm{C}$, et al. Necrotizing sarcoid granulomatosis with hemoptysis: a case report and literature review. Diagn Pathol 8: 79, 2013.

9. Chittock DR, Joseph MG, Paterson NA, McFadden RG. Necrotizing sarcoid granulomatosis with pleural involvement. Clinical and radiographic features. Chest 106: 672-676, 1994.

10. Koss MN, Hochholzer L, Feigin DS, Garancis JC, Ward PA. Necrotizing sarcoid-like granulomatosis: clinical, pathologic, and immunopathologic findings. Hum Pathol 11 (5 Suppl): 510-519, 1980.

11. Niimi H, Hartman TE, Muller NL. Necrotizing sarcoid granulomatosis: computed tomography and pathologic findings. J Comput Assist Tomogr 19: 920-923, 1995.

12. Arfi J, Kerrou K, Traore S, et al. F-18 FDG PET/CT findings in pulmonary necrotizing sarcoid granulomatosis. Clin Nucl Med 35: 697-700, 2010.

13. Mukhopadhyay S, Wilcox BE, Myers JL, et al. Pulmonary necrotizing granulomas of unknown cause: clinical and pathologic analysis of 131 patients with completely resected nodules. Chest 144: 813-824, 2013.

14. Furuya K, Shimizu R, Hirabayashi Y, Ishii R, Fukuda H. Stress hormone responses to major intra-abdominal surgery during and immediately after sevoflurane-nitrous oxide anaesthesia in elderly patients. Can J Anaesth 40 (5 Pt 1): 435-439, 1993.

15. Helmy SA, Wahby MA, El-Nawaway M. The effect of anaesthesia and surgery on plasma cytokine production. Anaesthesia 54: 733$738,1999$.

16. Statement on sarcoidosis. Joint Statement of the American Thoracic Society (ATS), the European Respiratory Society (ERS) and the World Association of Sarcoidosis and Other Granulomatous Disorders (WASOG) adopted by the ATS Board of Directors and by the ERS Executive Committee, February 1999. Am J Respir Crit Care Med 160: 736-755, 1999.

The Internal Medicine is an Open Access article distributed under the Creative Commons Attribution-NonCommercial-NoDerivatives 4.0 International License. To view the details of this license, please visit (https://creativecommons.org/licenses/ by-nc-nd/4.0/).

(C) 2018 The Japanese Society of Internal Medicine Intern Med 57: 1625-1629, 2018 